\title{
Body Plan Identity: A Mechanistic Model
}

James DiFrisco ${ }^{1}$ and Günter P. Wagner ${ }^{2,3,4,5}$

1) Institute of Philosophy, KU Leuven

2) Department of Ecology and Evolutionary Biology, Yale University

3) Yale Systems Biology Institute

4) Department of Obstetrics, Gynecology and Reproductive Sciences, Yale Medical School

5) Department of Obstetrics and Gynecology, Wayne State University, Detroit, MI

Corresponding author: gunter.wagner@yale.edu

JD ORCID: 0000-0001-9600-1013

GPW ORCID: 0000-0002-3097-002X

Abstract: A body plan is a stable configuration of characters for a major taxonomic group, such as chordates or arthropods. Despite widespread casual reliance on the concept for guiding comparisons within and between groups, the nature of body plans as well as the biological causes underlying their evolution have remained elusive. This paper proposes an abstract mechanistic model of body plan identity. We hypothesize that body plans are an evolutionary phenomenon that only applies to a relatively small subset of major clades, rather than being associated with each and every so-called "phylum." Body plans arise in evolution by stepwise accretion, and require a level of developmental complexity that is only found in some animal clades. Further, we suggest that, parallel to the developmental mechanisms controlling character identity, there are "body plan identity mechanisms" (BpIMs) that maintain entire configurations of characters while possessing a mechanistic architecture that is itself stable and traceable through evolutionary change. These BpIMs, we suggest, are entrenched intercellular signaling networks operating between transient embryonic structures that are destined to differentiate into distinct individualized characters. The activity of a BpIM results in a transient long-range integration of the embryo that is highly sensitive to genetic and environmental perturbations, and that can be detected morphologically as a conserved phylotypic stage. This model is illustrated with detailed interpretations of the notochord signaling system and the segment polarity network as candidate BplMs in vertebrates and arthropods, respectively. We conclude by contrasting the proposed developmental-mechanistic conception of body plans with the phylogenetic notion of ground plans, and sketch the general outlines of an empirical research program on body plan evolution.

Keywords: body plan, burden, phylotypic stage, hourglass model, notochord, segment polarity

Acknowledgments: GPW gratefully acknowledges the financial support of the John Templeton Foundation (grant number 61329). The opinions expressed in this paper are those of the authors and not those of the JTF. JD thanks the Research Foundation - Flanders (FWO) for financial support (grant number 41277 and 88559). 
"We surmise that the commonality of anatomy among groups sharing a body plan is not merely an incidental sharing of descriptive features, but rather, that those features are the component parts of a deeply integrated shared pattern of development."

- $\quad \operatorname{Raff}(1996$, p. 31)

\section{Introduction}

The "body plan" is a central organizing concept for understanding metazoan evolution and diversity. It arose as a phenomenological concept based on the recognition that major groups of animals have fundamentally similar configurations of characters in spite of their great variety of lifestyles and special adaptations (Nyhart, 1995; Rupke, 2009). It is paralleled by special patterns of conservation during development known as phylotypic stages (Sander, 1983; Slack et al., 1993). In this paper we aim to pick up a line of thought that was proposed by early pioneers of evolutionary developmental biology such as Rupert Riedl (1975), Brian Hall (1992) and Rudy Raff (1996), and seek to articulate this theoretical stance in the light of recent developments in comparative developmental biology and evolutionary biology.

A curious evolutionary feature of body plans is their entrenchment: once they evolve, further evolutionary change seems to be largely restricted to modifications of that basic design rather than transformation into a novel or existing body plan, except in cases of major reduction in body complexity such as in parasitism (e.g., some parasitic groups such as Acantocephala and Rhizocephala). This striking phenomenon is classically framed as a problem of understanding why the major animal body plans have remained largely static since roughly the Cambrian ( 550 mya), despite the vacant ecological niches opened up by mass extinctions and terrestrial invasion (Raff, 1996; Erwin et al., 1987; Valentine, 2004). This problem has dominated evolutionary discussion of body plans, though we will see reasons to think that major novel body plans have appeared since the Cambrian (e.g., Holometabolan larvae). In any case, there is much more to the concept than a puzzling evolutionary pattern to be explained. We interpret the classical body plan concept as a pointer to an important evolutionary phenomenon, and we attempt to describe what this is.

To see how the body plan is not only a pattern but also something that can explain evolutionary patterns, it is instructive to consider how entrenchment is a likely consequence of the evolution of complexity at any level. In an evolving system, novel traits can arise that developmentally or functionally depend on existing traits. When those novelties are adaptively maintained, the prior traits they depend on experience additional evolutionary constraints with respect to modifications that would disrupt the development of the novel traits. Over time, this process leads to the evolutionary fixation of those traits that newer traits depend on, called "burden" (Riedl, 1978) or "generative entrenchment" (Wimsatt, 1986). The establishment of DNA and RNA as replicating template macromolecules in terrestrial life is perhaps the most extreme example of an entrenched biological trait. Importantly, these fixed deposits of evolution are sources of generalizations and regularities that cut across the variations that accumulate from further evolution. The success of molecular biology, for example, depends on the universality that comes from the evolutionary entrenchment of basic molecular mechanisms. Body plans are similarly entrenched deposits of evolution at the level of whole-organism metazoan development. Their evolutionary conservation 
confers significant "projectibility": knowing that some organism is a vertebrate provides abundant information and predictability about its development and structure even if it is otherwise unknown (Riedl 1978).

In this paper, we develop a concept of body plans as an evolutionary phenomenon that can occur given a degree of complexity of body organization, which makes entrenchment likely to happen. The evolutionary phenomenon is the "reification" of a configuration of characters and developmental traits that is shared within a clade or a paraphyletic assemblage. We do not assume that body plans are identified with particular taxonomic levels or categories, such as socalled phyla, nor that each and every major clade displays a distinct body plan. Instead, the question whether a particular clade exhibits a body plan in the relevant sense is an empirical question-not one that automatically follows from the definition of a clade or phylum.

Thinking of body plans not only as macroevolutionary patterns but as entrenched reservoirs of high-level regularities about major animal groups elevates the promise and payoff of reaching a better understanding of their nature. This more robust notion of body plan is particularly important for comparative biology. Only a small sample of model organisms out of the vast diversity of life can be studied in depth. Researchers need principled means for determining how to project or extrapolate their results from model to non-model organisms, including humans, thereby amplifying the inferential richness of the limited knowledge we in fact have. The body plan can be thought of in this way as a road map of projectibility that systematizes knowledge about organismic development across diverse species.

This positive role for the concept is significantly enhanced by mechanistic models of the interdependencies in development that give rise to evolutionary conservation of body plans. Such models would contribute not only to explaining macroevolutionary patterns of body plan conservation, but also to understanding the possibilities and constraints on the evolvability of specific body plans, while also refining and clarifying the body plan concept. With this end in view, we propose a conceptual model of body plan identity mechanisms, building on the framework of character identity mechanisms (DiFrisco et al., 2020; DiFrisco et al., in press) together with work on the developmental hourglass model from Raff (1996), the burden concept of Riedl (1978), and others. This model leads us to propose a developmental-mechanistic body plan concept in addition to the phylogenetic one. Viewed phylogenetically, a body plan is roughly a set of characters that characterize a monophyletic group. By contrast, in the model we propose, a set of characters can characterize a monophyletic group because they are integrated by the same body plan identity mechanism.

\section{Body plan identity versus state}

Organisms with very different outward morphologies can nonetheless share the same body plan, such as whales, seahorses, and giraffes. The body plan of these vertebrates is an abstract notion rather than a directly observable or measurable feature like size or shape. In this respect body plans are like homologies, but for whole organisms. The tetrapod forelimb is not identical to any particular wing, leg, or fin with determinate biometric features, but instead captures what is evolutionarily conserved across all tetrapod forelimbs. 
If body plans are not amenable to direct observation or measurement, there is a need to specify how they can be characterized and identified. Such a clarification is also needed for illuminating the relationships between body plans and an array of related concepts, including the phylotypic stage, archetype, group ancestor, and cladistic ground plans. ${ }^{1}$

Following the parallel with homology, we propose that there is a biologically meaningful distinction between body plan identity and body plan state, parallel to the distinction between character identity and character state (Wagner, 2014). The character identity of the tetrapod forelimb refers to its identity as a body part, which can exist in many different character states in particular tetrapod lineages-e.g., as wings, legs, or flippers. Character identity is based on the relative position of the character within the body plan as well as the other operational criteria traditionally used to establish homology, such as "special quality" and the presence of transitional forms (Remane, 1952; see Wagner, 2014; Fusco and Minelli, 2013; McKenna et al., 2021). By contrast, character states include properties of the body part, such as its size, biometric shape, color, its number of iterated sub-parts, and so on. Wagner $(2007 ; 2014)$ proposed that character identity possesses a distinguishable developmental-genetic basis from the mechanisms responsible for different character states, called "Character Identity Networks" (ChINs) or "Character Identity Mechanisms" (ChIMs) (DiFrisco et al., 2020). In addition, in this model, character identity is not determined immediately in early development, but only appears once the ChIMs are active.

Body plan identity can be conceptualized along similar lines, as the set of morphological character identities and their interrelationships that characterize a major clade. Like character identity, body plan identity is not determined immediately in early development, but first appears around a conserved mid-developmental stage known as the so-called "phylotypic stage" (Raff, 1996; Duboule, 1994; Slack et al., 1993). Body plan states consist of the specific realization a body plan assumes in particular species and lineages. Body plan states manifest themselves in the character states realized by their constitutive parts. For example, whales and giraffes both possess vertebrae (character identity) but they have very different shapes and sizes (character state). Vertebrae are part of the vertebrate body plan, but determinate shapes and sizes of vertebrae are only specific states that the body plan can take. In addition, body plan states can include novel derived states that evolved in sub-clades after the crown group ancestor, but are not integrated parts of the body plan. For instance, hair and mammary glands are found only in mammals, and thus characterize a subset of vertebrate species.

This distinction between identity and state allows us to see why some existing methods for measuring body plan conservation are misguided. In an often-cited study, Richardson et al., (1997) conducted a comparative quantitative anatomical assessment of the vertebrate phylotypic stage, around the tailbud stage when somitogenesis is largely finished. They found anatomical differences in vertebrate embryos in features like number of somites, number of pharyngeal arches, size, and heterochronic shifts such as rate of somitogenesis. They concluded from this that, contrary to the predictions of the hourglass model (Raff, 1996), "there is no highly conserved embryonic stage in the vertebrates." The conclusion does not follow, however, because almost all of the traits used in their study to measure body plan conservation are character states rather than character identities. Compare the claim that there is no conserved avian wing because

\footnotetext{
${ }^{1}$ We do not distinguish between concepts of body plan and Bauplan, which we understand to be equivalent.
} 
particular avian wings have different sizes, proportions and numbers of feathers. To assess body plan identity, conservation should be evaluated at the more abstract level of morphological homology, or character identity, rather than morphometrics of character states. A similarly problematic assessment of the phylotypic stage was published about zebrafish development (Schmidt and Starck, 2011).

The identity versus state distinction also aids in clarifying the relationship between body plans and related concepts with which it is sometimes confused. The body plan is not the same as the phylotypic stage because adults also share body plans, though it has long been recognized that adults often have the most divergent body plan states (von Baer, 1828; De Beer, 1954). The phylotypic stage is just the developmental interval when the body plan features first appear, but a particular vertebrate at this stage is still in a determinate body plan state, having, e.g. a specific size and number of somites.

Although abstract, the body plan is not the same as the idealistic "archetype" concept of Owen and other pre-Darwinian rational morphologists. The difference is that the body plan is an evolutionary and variational concept: body plans arise gradually in evolutionary history by stepwise accretion of interdependent characters, and they have a spatiotemporally restricted evolutionary career. The loss of entrenched body plan characters is unlikely (though not impossible), whereas new characters can continue to be integrated into existing body plans by the same accretion process, yielding a natural hierarchy of ancestral and derived body plans. The body plan is thus not the "archetype," but neither is it identical to the group ancestor in which it first emerged-e.g., the first vertebrate-any more than the tetrapod forelimb is identical to the pectoral appendage of the first tetrapod. The ancestor is one particular state that a body plan can take, among others.

A more subtle question is whether body plans can be identified with sets of characters shared in a monophyletic group. There are two versions of this idea. One longstanding thesis, etched in the term "phylotypic," holds that metazoan body plans are conserved at the phylum level, with each phylum having its own distinct body plan. However, taxonomic ranks above species are agreed to be arbitrary or conventional (Mayr, 1970; Eldredge and Cracraft, 1980), and thus it is biologically meaningless to link a concept like body plan to a particular Linnean category. There may also be subphylum body plans: for example, a distinct body plan for Holometabola within the "phylum" Arthropoda (see Section 5 below). The taxonomic rank at which significantly distinct body plan identities are to be found is an open issue to be discovered. An envisioned payoff of understanding the mechanistic architecture underlying body plan identity is to aid in identifying and explaining where in phylogeny novel body plans arose.

A second, more sophisticated version is the cladistic concept of the "ground plan" or "ground pattern" (Hennig, 1965; Ax, 1984; Yeates, 1995; Scholtz, 2004). The ground plan is a set of characters describing the stem species of a monophyletic clade, or the crown group ancestor (Budd and Jensen, 2000), which includes a combination of ancestral plesiomorphies and derived apomorphies. Without any biological restrictions on cladistic formalism, however, the nestedness of phylogenetic trees implies that there is a ground plan for every monophyletic group, however trivial their differences of body organization may be. There is no question that cladistic methods are useful for mapping phylogenetic relationships, but on their own they do not provide a biologically informative basis for a body plan concept. 
Because the identity-state distinction is reflected in the underlying developmental architecture of morphological characters, perhaps the same holds for integrated ensembles of morphological characters, e.g. body plans. We hypothesize that this is the case, and that there are "body plan identity mechanisms" (BpIMs), active in mid-development, that are distinguishable from the mechanisms influencing states of characters or of body plans. In this model, BplMs are based on entrenched interactions between signaling centers that organize the body of the embryo into a specific configuration of characters, like character identity mechanisms for organs, with some key differences. BpIMs are hypothesized to explain the puzzling evolutionary conservatism of body plans (see Introduction), while also aiding identification of novel body plan identities. The BpIM hypothesis also serves the aim of bringing mechanistic insights to the high-level regularities about major animal groups embedded in the body plan concept.

\section{Identity mechanisms from body parts to body plans}

Evidence for the distinct developmental control of character identity comes from a variety of sources. The past decades of comparative developmental genetics has revealed extensive variability in the components and processes underlying homologous characters, a phenomenon known as developmental system drift (True and Haag 2001; Haag and True 2018; Hall 1995). At the same time, the same studies have also uncovered extensive conservation of key developmental regulators. This has been found even in cases where the homology of the overlying characters is doubtful, such as eyes and limbs of distantly related metazoans - a phenomenon known as "deep homology" (Shubin et al., 1997, 2009; see DiFrisco et al., in press). Close examination of these contrary patterns reveals that the evolutionary variability and conservation are not uniformly distributed across all developmental control mechanisms for a body part (Wagner 2007). Instead, there are causal "bow-tie" or "hourglass" patterns in which core modules are activated by variable inputs and can switch on variable outputs. This has been documented in cell biology studies showing that a small number of highly conserved control genes regulate the expression of more labile realizer genes determining the cell phenotype (Hobert 2008, 2011; Arendt et al., 2016; Almeida et al., 2021). At higher levels of morphology, an especially clear example concerns the insect homeobox gene Ultrabithorax (Ubx). Knockdown of Ubx in Tribolium results in development of forewing identity (elytra) in the position of the hindwing, indicating that the gene functions as a simple modular switch for a complex cascade of developmental processes of wing morphogenesis (Deutsch 2005; Wagner 2007, 2014; Tendolkar et al., 2021). In this sense, Ubx controls hindwing character identity, as opposed to the phenotypic state of the wing (e.g. a wing blade or a haltere). The difficulty, of course, is that a loss of function mutation of Ubx or any other character identity gene does change the phenotype of the appendage, but the Tribolium example clearly shows that it is the character identity that is affected not just the shape of the hindwing because there is no insect in which the hindwings are shaped as elytra.

Synthesizing these findings, the idea of "character identity networks" (ChINs) (Wagner 2007; 2014) was proposed as a model for the gene-regulatory control of character identity. ChINs are gene regulatory modules that mediate between signaling input and the genetic mechanisms controlling execution of the phenotype. The structure of the ChIN was conceptualized as 
consisting of regulatory genes, with internal cross-regulatory interactions that sustain each other's expression and collectively switch on batteries of effector genes for a specific character, while also repressing the expression of genes that initiate alternative developmental fates. The combination of ChIN genes being individually necessary for each other's expression, and acting collectively to regulate effector processes, makes the ChIN especially refractory to evolutionary change. By contrast, the inputs activating the ChIN are thought to act in a perturbative and redundant fashion, making them more evolutionarily labile. Similarly, the outputs or realizer gene batteries are responsive to shifting selection regimes, for example transforming a wing into a flipper (e.g. penguins), and so are expected to diverge in different lineages over evolutionary time. The differential evolutionary stability of these stages of developmental mechanism is connected to the broader biological role of ChINs. This role is to activate developmental processes within specific domains of the embryo, "individualizing" the resultant character from other characters and supporting their long-term evolvability. The modularity of ChINs vis-a-vis other mechanistic stages allows them to stably play this role without being caught up in their evolutionary dynamics.

Further developments of this framework showed how the ChIN model, which is based on transcriptional gene regulatory networks, is just one realization of a wider category of "character identity mechanisms" (ChIMs) (see DiFrisco et al., 2020). In the present context, the key novelty of the ChIM model is to hypothesize that there are different mechanisms, with different components and activities, for character identities at different levels of anatomical organization (see Table 1). Cell type identities can be determined by Core Regulatory Complexes (CoRCs) of transcription factors (Arendt et al., 2016). Tissue ChIMs, by contrast, have cell types and ECM as their components, and are controlled and maintained by cell-cell signaling activities. Finally, organ ChIMs are signaling centers and activities that control the spatial arrangement of tissues in a developing organ rudiment. These different ChIMs each represent parts of the total developmental mechanism influencing development of an anatomical part, and each is hypothesized to possess a similar, ChIN-like "causal profile."

\begin{tabular}{|l|l|l|l|}
\hline $\begin{array}{l}\text { Anatomical } \\
\text { Unit }\end{array}$ & $\begin{array}{l}\text { Control outcome achieved } \\
\text { by ChIM }\end{array}$ & Parts of the ChIM & Activities of the ChIM \\
\hline Cell type & $\begin{array}{l}\text { Reaction norm in response to } \\
\text { signals and other } \\
\text { environmental stimuli } \\
\text { Enabling differential gene } \\
\text { expression }\end{array}$ & $\begin{array}{l}\text { Gene networks (ChINs) } \\
\text { TF complexes (CoRCs) } \\
\text { non-coding RNA }\end{array}$ & $\begin{array}{l}\text { Cross-regulatory } \\
\text { activation or repression } \\
\text { Autocrine signaling }\end{array}$ \\
\hline Tissue type & $\begin{array}{l}\text { Local environment of cell } \\
\text { types and ECM within tissue }\end{array}$ & $\begin{array}{l}\text { Cell types } \\
\text { ECM } \\
\text { Signaling molecules }\end{array}$ & $\begin{array}{l}\text { Cell-cell signaling } \\
\text { ECM production }\end{array}$ \\
\hline Organ & $\begin{array}{l}\text { Spatial arrangement of } \\
\text { tissues in a specific domain of } \\
\text { the embryo }\end{array}$ & Signaling centers & $\begin{array}{l}\text { Mutually } \\
\text { interdependent } \\
\text { signaling activities } \\
\text { among } \\
\text { centers signaling }\end{array}$ \\
\hline
\end{tabular}

Table 1. Character identity mechanisms (ChIMs) for anatomical units at different levels of organization (see text for details). 
The causal profile characterizing ChIMs includes a number of features (DiFrisco et al., 2020). Most importantly, they are actively modular, with components that maintain or positively feed back on one another, and that negatively inhibit alternative regulatory states. Components of the ChIM are interdependent, i.e. necessary for each other's functioning, and also collectively necessary for development of the target character (e.g., Ubx and hindwing identity). This developmental role of ChIMs is causally non-redundant in the sense that other causes do not have the same effects. This is contrasted with the genetic influence on polygenic traits such as body size, which is characterized by high redundancy and aggregativity.

Together, these developmental features have a number of evolutionary consequences (DiFrisco et al., 2020). ChIMs are less replaceable than their upstream signaling inputs and downstream effector mechanisms. They are therefore more evolutionarily conserved, and can be traced across individuals and species along with the relevant character identity. The features of interdependence and necessity make ChIMs more heavily entrenched or burdened than other developmental mechanisms. Following the discussion of entrenchment (see Introduction), ChIMs are also a source of potentially surprising, wide-ranging generalizations across evolutionary lineages (see DiFrisco et al., 2020). These features, of course, do not mean that these mechanisms cannot change in evolution. The greatest potential for this is after gene or genome duplication, which can generate redundancies where there were previously none, and lead to some genetic change compatible with their causal role.

Supposing that individual morphological characters possess this underlying developmental architecture, then, is there a similar architecture for entire body plans? The ChIM model of morphological homology shares key attributes with the developmental hourglass model of body plan conservatism - particularly, the three stages with a conserved middle stage characterized by high developmental burden. An intriguing question is whether the same causal profile (active modularity, interdependence and necessity, and non-redundancy) can be detected in mechanisms operating during the phylotypic stage-the narrow interval of the hourglass. In the following section we will argue that this is likely the case.

\section{A model of body plan identity mechanisms}

\subsection{The hourglass model: variational properties of different developmental stages}

Generative entrenchment describes an evolutionary process in which ancestral characters become increasingly entrenched as new characters evolve that depend on them. Stated in the abstract, this class of models (Riedl 1978; Wimsatt 1986; Arthur 1988) would predict greater conservation of earlier stages in development and increasing divergence in late development, along the lines of von Baer's laws of embryology. What is actually observed, however, is a surprising divergence of early development, followed by, in some lineages, a highly conserved mid-development, leading to an expectedly divergent late development. This is the pattern captured by the "hourglass model" (Sander 1983; Elinson 1987; Duboule 1994; Raff 1996). The key insight that brings the entrenchment models into conformity with the hourglass pattern is that the developmental dependencies causing entrenchment have different variational properties at different stages of development. 
Early development, extending roughly from fertilization to gastrulation, is characterized by global patterning via axial gradients and by shape regulation. Many processes at this stage exhibit developmental and evolutionary flexibility of pathways together with robustness of morphogenetic outcomes. This is illustrated most clearly in gastrulation, the process that transforms a hollow ball of cells (blastula, or equivalent stage in other embryos such as the inner cell mass of the blastocyst of eutherian mammals), into an embryo (gastrula) with distinct germ layers. Metazoans exhibit a striking diversity of modes of gastrulation, variously involving ingression, invagination, epiboly, and delamination. Germ layers also develop out of different locations on the animalvegetal axis of the egg in different groups (Favarolo and López, 2018). Yet the germ layers ultimately converge on similar topological relationships later in the embryo. There are many ways, genetically and morphogenetically, to arrive at the same important early to mid-developmental stages. This permits adaptive divergence in early development under different life history strategies, such as variations in egg and yolk size or placentation over several orders of magnitude and the evolution of placentation.

Later development, which in bilaterians can be marked from the time when organs are proceeding to their definitive individuated forms, is characterized by a more modular organization of the embryo. Here, development takes place quasi-independently in distinct regions, in contrast to the global patterning characteristic of early development. Eventually, more global coordination of development is handed over to the neural-endocrine system, which regulates growth and shape changes of characters that are already established. The modular organization of late development allows for high evolutionary divergence under natural selection since it allows characters to vary quasi-independently from one another (Lewontin 1978; Wagner 2014).

Mid-development, which extends roughly from gastrulation through organogenesis (depending on the clade), mediates between early and late modes of embryonic organization. According to Raff's (1996) hourglass model, mid-development "exhibits a high interconnectivity between elements that will later come to represent separate modules" (p. 204). Specifically, inductive signaling interactions take place between tissues that are destined to later develop quasi-autonomously, such as the heart mesoderm helping to induce parts of the eye in vertebrates (ibid). These signaling interactions are highly entrenched: later development depends on them, thus they put constraints on morphological evolvability at that mid early stage. The conservatism of phylotypic stages like the vertebrate pharyngula is hypothesized to be explained in this way.

Recently, a number of studies have confirmed the hourglass model of differential conservation at the transcriptomic level. The first tests of the hourglass model that go beyond the morphological facts of mid-developmental conservation were based on transcriptomic data, showing that gene expression divergence between species is least at the time of the phylotypic stage (Irie and Kuratani, 2011), which interestingly was done first not in vertebrates but in Drosophila (Kalinka et al., 2010), suggesting that phylotypic stage conservation is not limited to vertebrates. Tests that extend to the genomic level compared the molecular genomic divergence of genes expressed in different stages of development. In 2005, Hazkani-Covo and colleagues showed that stabilizing selection of genes expressed in the phylotypic stage is stronger than on genes expressed in other stages (Hazkani-Covo et al., 2005). Among studies of this kind we highlight the quite comprehensive study by Piasecka and collaborators (2013). In that study the authors have shown that genes expressed in the phylotypic states of mouse and zebrafish have a high frequency of 
old "bilaterian" genes, a high fraction of transcription factor genes associated with highly conserved non-coding sequences as well as transposon free regions (both suggesting high degree of cis-regulatory conservation). The most direct test that perturbations during the phylotypic stage are more likely harmful than during other stages of development, a core assumption of Raff's hourglass model, was provided by Galis and Metz in a meta-analysis of studies of embryo-toxicity of retinoic acid (Galis and Metz, 2001). Galis and Metz showed that administration of retinoic acid to pregnant females of hamsters, mice and rats had the highest impact on neonate mortality and morbidity during the pharyngula stage (the phylotypic stage of vertebrates). A study of effects of perturbation on the segmented germband stage in Drosophila, which has been proposed as the arthropod phylotypic stage, showed similar vulnerability (Galis et al., 2002). All of this evidence shows that the conservation of the early to mid developmental stages is not an artifact of morphology but a real pattern of preferred evolutionary conservation.

While these studies have provided valuable experimental evidence that the hourglass pattern is real and caused by strong selection against changes in that stage of development, two issues remain. On the one hand, it is not clear what interactions cause the conservation of the phylotypic stage as described in Raff's model. On the other hand, the implications of the phylotypic stage have not been much discussed in terms of its implications for the body plan concept. To these questions we want to turn next.

\subsection{Body plan identity mechanisms}

An instructive starting point is to consider the developmental role of signaling between germ layers. Germ layers represent a primitive, early state of differentiation in the progression of embryogenesis toward a more complex, differentiated, and modular state. After gastrulation, germ layer tissues are only weakly "individualized" with respect to their developmental fates (Row et al., 2016). Single germ layers have been shown experimentally to lack capacities for complex development. In chick embryos, for example, signaling from the notochord (mesoderm) is necessary to initiate pancreas development (endoderm) as well as neural tube differentiation (ectoderm) (Grapin-Botton and Melton, 2000). Signals from cardiac mesoderm are necessary to turn on endodermal liver markers (Gualdi et al., 1996). Generally, regionalization of the endoderm requires interaction with the gut mesoderm, as in Drosophila (Grapin-Botton and Melton, 2000; Bienz, 1997). Germ layer origin also does not strongly restrict the developmental capacity of a cell population, as shown by transplantation experiments in mice in which cells fated to one germ layer adopt the fate of the surrounding tissue after transplantation (Tam and Gad, 2004). This developmental flexibility is reflected by phylogenetic variation in the germ layer origin of homologous organs, which spelled the demise of the germ-layer theory of homology from classical embryology (de Beer, 1954).

More highly conserved than germ layer origins are the inductive signaling interactions between germ layers. It is not the origin of a rudiment in a germ layer that confers a capacity for complex development, but the accessibility of interfaces between germ layers and epithelialmesenchymal surfaces that permit inductive signaling. Long-range signaling interactions across germ layers thus seem to be necessary to progress out of the post-gastrulation state of differentiation to the regional quasi-independence characteristic of later development. These observations are key for a deeper understanding of the phylotypic integration hypothesized by 
the hourglass model. "Integration" in this context should be understood as causal interdependence between parts for their development and maintenance -i.e., the opposite of modularity-and not only as statistical covariance. When interaction across weakly individualized embryonic structures like germ layers is necessary for their further differentiation into organs and organ systems, then the differentiation of the embryo into quasi-autonomous characters must be based on a prior developmental integration of the whole embryo. This suggests that the high integration characteristic of phylotypic stages is not only a product of constraints on variability, but also has the positive biological role of allowing complex modular characters to functionally differentiate and specialize.

The inductive interactions between germ layers in mid-development are based primarily on major signaling pathways such as Shh, Wnt, and BMPs. The signaling centers and signaling activities tend to act interdependently and synergistically to induce the differentiation of adjacent embryonic structures. We refer to these as body plan identity mechanisms (BpIMs) due to their role in maintaining body plan identity in the sense of an evolutionarily conserved configuration of homologous parts. BpIMs are mechanistically similar to character identity mechanisms for whole organs such as the limb or the brain: organ ChIMs are signaling centers whose signaling activities control the spatial distribution of tissues within an organ rudiment (DiFrisco et al.,. 2020). The major difference is that the tissue domains covered by the signaling activities of organ ChIMs are destined to be integral parts of the same individualized character, whereas the tissue domains covered by the signaling activities of BpIMs are multiple, weakly individualized embryonic precursors of distinct organ systems. The integration of embryonic structures exhibited by the phylotypic stage can be explained in terms of the integration of an underlying body plan identity mechanism. To illustrate this abstract conceptual model, we turn to a well-studied candidate BpIM within the chordates-signaling interactions between embryonic structures surrounding the notochord.

\subsection{The notochord signaling system is (part of) a BpIM}

The notochord is an embryonic character found in the embryos of chordates including urochordates, and vertebrates, and is a synapomorphy of the chordate clade. First described by von Baer, the notochord is a mesodermal rod of rigid tissue that develops out of the dorsal organizer during gastrulation, and runs along the rostrocaudal length of the dorsal midline, ventral to the neural tube (see Fig. 1). Originally, it likely functioned to enable undulatory locomotion by providing a hydrostatic support for the contraction of longitudinal muscles (Annona et al., 2015; Long et al., 2002). Some non-vertebrate chordates (amphioxus and appendicularian tunicates) and a few vertebrates (hagfishes, lampreys, and sturgeons) keep the notochord through adulthood (Annona et al., 2015), suggesting that it retains its skeletal-locomotory function in these groups. In all other vertebrates, the notochord is only present in the embryo (or larval stage in amphibians) and gets replaced later in development by the spinal column. In these vertebrates, the function of the notochord seems to be purely developmental: it is an essential, highly entrenched source of signaling molecules that induce differentiation of surrounding tissues. For this reason, the notochord is a paradigmatic body plan character (Wagner and Laubichler 2004; Raff 1996), and the notochord signaling system can be investigated as part of a potential body plan identity mechanism (BpIM) for vertebrates. 
Many ectodermal structures depend on signals from the notochord (mesoderm) to differentiate (see Fig 1). A signaling pathway with many crucial roles is Sonic Hedgehog (Shh). In amniotes, Shh secreted from the notochord is necessary and sufficient for inducing formation of the floor plate as well as the ventral neural tube (Ribes et al., 2010). Shh secreted from the notochord and floor plate also acts as a morphogen gradient for dorso-ventral and mediolateral differentiation of somites (mesoderm). Low Shh levels together with Wnt signaling from the roof plate induce dermomyotomal gene expression (Pax3), and slightly higher Shh levels instead activate myogenic differentiation (Myf5 and MyoD). Closer to the notochord, high Shh levels together with the notochord-secreted BMP antagonist noggin induce sclerotomal gene expression (Pax1) instead of myotomal markers (Corallo et al., 2015, p. 2997). This Shh gradient emanating dorsally from the notochord interacts with an anti-parallel gradient of Wnt and BMP diffusing ventrally (Briscoe and Small 2015). In the neural tube, Wnt and BMP promote dorsal neural identities, and dermomyotomal identities in the somites. The opposed gradients can also repress each other-for example, Wnt activates Gli3 and Gli3- $R$ which represses Shh activity in the dorsal neural tube, and Shh inhibits the expression of dermomyotomal marker Pax3 (Le Dréau and Martí, 2012; Corallo et al., 2015).

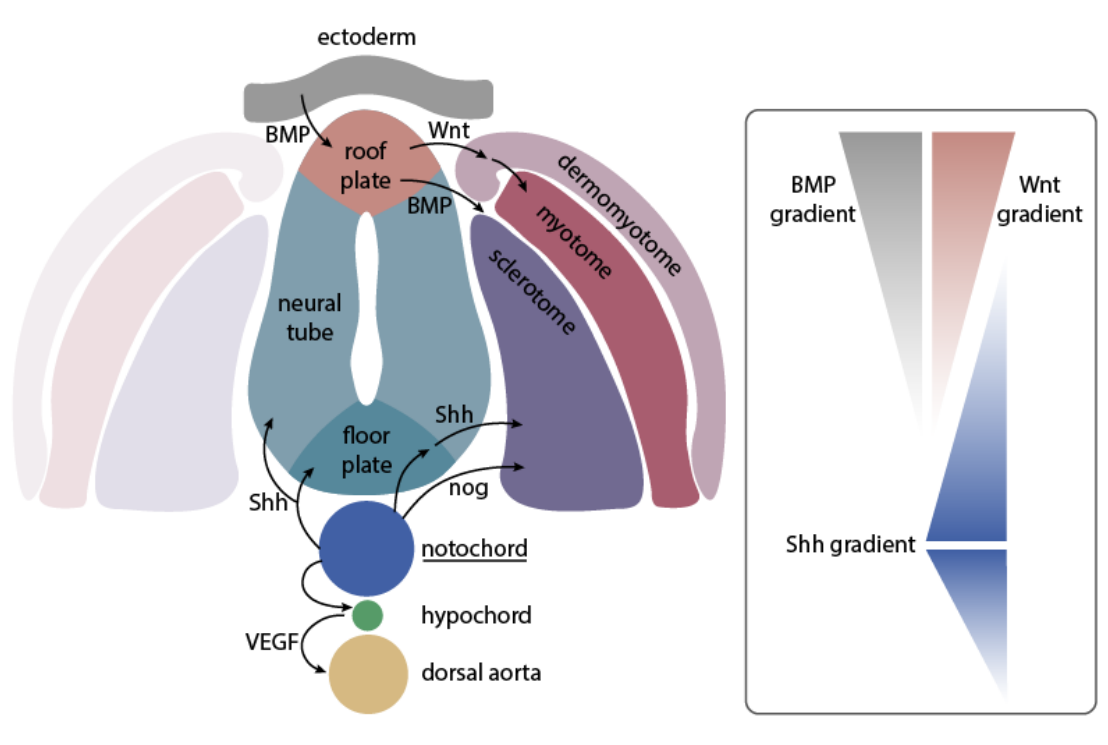

Fig 1. Key components of the notochord signaling system, part of a proposed body plan identity mechanism (BpIM). In the transverse section of axial structures shown here, inductive interactions across embryonic tissues are influenced primarily by dorsoventral gradients of signaling molecules Shh, BMP, and Wnt.

The notochord is also a source of inductive signals for morphogenesis and differentiation of endodermal and mesodermal structures. In chick embryos, activin- $\beta B$ and fibroblast growth factor 2 (FGF2) secreted from the notochord permit pancreatic morphogenesis by repressing endodermal Shh (Hebrok et al., 1998). Lower vertebrates possess another transient body plan character-the hypochord - which is a line of endodermal cells situated immediately ventral to the notochord. During early neurulation, signals from the notochord are required to initiate hypochord development. Experiments on Xenopus showed that the hypochord secretes vascular endothelial 
growth factor (VEGF), which directs the migration of angioblasts to the dorsal midline where they form the dorsal aorta (Cleaver and Krieg, 1998). Prior to this, notochord-derived noggin and chordin actively inhibit midline vascularization until the paired dorsal aorta can fuse (Corallo et al., 2015). Other effects of notochord signaling in vascular development include cardiac left-right asymmetry, control of arterial versus venous identity of major axial blood vessels, and specification of endocardial progenitors in zebrafish (Stemple 2005; Corallo et al., 2015). Shh ligands from the notochord and floor plate are also required for proper positioning of the kidney primordia (Tripathi et al., 2010; Corallo et al., 2015).

These examples illustrate how many essential and entrenched signaling interactions across germ layers surround just one embryonic structure in mid-development. Notochord-derived signaling represents just a sample out of the wider ensemble of signaling interactions that make up the entire identity mechanism for the vertebrate body plan (BpIM), in particular the arrangement and identity of the so called axial organs. Although it is a particularly entrenched character, the notochord itself develops by inductive signaling from other tissues in reciprocal fashion. In zebrafish, it is induced by Wnt signaling within a population of bipotential notochord/floor plate cells, and is differentiated from the hypochord by Notch signaling within a population of bipotential hypochord/notochord cells (Row et al., 2016). The notochord is thus a part of a larger interdependent signaling network involving all germ layers-a body plan identity mechanism-which is essential for setting up the developmental capacity for regional differentiation of distinct characters in late development.

\subsection{Toward a causal profile of body plan identity mechanisms}

Situating experimental studies of notochord signaling from model systems in a comparative context can start to reveal the relative variability or entrenchment of different elements of the system. Interestingly, signaling interactions often seem to be more highly conserved than the identity of the embryonic structures acting as signaling centers, in line with the developmental flexibility of germ layers noted earlier. For example, amniotes lack a hypochord, but the role of the hypochord in patterning the surrounding vasculature appears to be played by the dorsal gut endoderm, which expresses VEGF near the same blood vessels (Dumont et al., 1995; Row et al., 2016). Multiple signaling centers can secrete the same signaling molecule. Hypochord, notochord, and floor plate express many of the same signals, such as Shh and VEGF, and ectoderm and roof plate both secrete BMP (Corallo et al., 2015; Ribes et al., 2010). The conserved role of interdependent signaling centers and pathways conforms to the model of organ ChIMs (DiFrisco et al., 2020). This suggests that identity mechanisms for body plans may possess the same "causal profile" characteristic of identity mechanisms for body parts, including the features of active modularity, complex organization, interdependence and necessity, and non-redundancy.

A mechanism is actively modular if its components maintain or positively feed back on one another and negatively inhibit alternative regulatory states. This feature is most well-documented in transcriptional networks controlling cell types (Hobert 2011; Arendt et al., 2016; Almeida et al., 2021), but it also seems to be present in the notochord signaling system. For example, after induction by notochord-derived Shh, the floor plate amplifies the signal with more Shh, and the hypochord secretes VEGF in a similar fashion (Ribes et al., 2010; Corallo et al., 2015). Notochordderived noggin and chordin exclude alternative cell identities (endothelial cells) in the midline prior 
to fusion of the dorsal aorta, and the boundaries between somite tissues depend on a series of repressive regulatory interactions responding to concentrations of Shh, Wnt, and BMP (Corallo et al., 2015). Although they seem to exhibit the positive and negative regulatory interactions characteristic of ChIMs, however, BpIMs are not modular in the spatial sense of occupying a restricted region of the embryo where an individual character will form. Instead, BpIMs are signaling networks distributed throughout much of the developing embryo.

It is clear that the notochord signaling system exhibits complex organization, meaning that heterogeneous components are organized in space and time to yield dynamics that are not the cumulative consequence of the activities of individual molecules. Although signaling molecules like Shh are often attributed causal responsibility for inductive signaling outcomes, in reality they depend on complex receptors, transduction machinery, and downstream GRNs. The importance of spatial organization in inductive processes is obvious, but timing or temporal organization is also crucial (Ebisuya and Briscoe, 2018). In amniotes, Shh signaling can induce floor plate identity from gastrulation to early somitogenesis stages but not later (Ribes et al., 2010), indicating that the cellular competence to respond to induction is just as necessary as signal secretion for induction to occur.

Another aspect of the causal profile of ChIMs is that strong interdependence between parts makes them individually necessary for each other's operation, as well as collectively necessary for the development of the character (see DiFrisco et al., 2021). In the notochord signaling system, likewise, major pathways depend on each other to pattern embryonic structures, for example, somitic differentiation via the coordinated activity of Shh, Wnt, and BMP. As a result, most of the major interactions of the notochord signaling system described above are necessary for the vertebrate body plan to develop. Lack of notochord-derived signals is generally catastrophic for development of subsequent structures without compensatory artificial interventions.

A factor's being causally necessary for some effect is often connected to non-redundancy: other components do not have the same effects that can compensate for experimental or evolutionary perturbations. In the notochord signaling system, this feature seems to apply more to the signaling activity than to the anatomical structures acting as signaling centers, though whether that is true of BpIMs generally is an open question. As noted, in different species multiple signaling centers can secrete the same signal, and different structures can play the role of signaling centers (e.g., hypochord in anamniotes versus dorsal gut endoderm in amniotes). When tissues induced by a signal start producing that same signal, it can create causally redundant signaling centers. For example, a study on mice carrying a mutation that caused premature degeneration of the notochord found that Shh from the floor plate was sufficient to support the differentiation of somites into sclerotome and vertebrae in the absence of a notochord (Ando et al., 2010). Notochord-secreted Shh was still necessary for prior induction of the floor plate, however.

In addition to these four features generic to the causal profile of all ChIMs, there is another important attribute that applies especially to identity mechanisms based on signaling networks (i.e., for organs and body plans). The concept of "kernels" from Davidson and Erwin (2006) describes core regulatory circuits of transcription factors and associated cis-regulatory sequences that are hypothesized to underlie the conservation of phylum-level body plans as well as body parts. In the ChIM model, kernel-like circuits are more appropriately viewed as identity mechanisms for cell types, whereas identity mechanisms for larger anatomical units are based 
on cell-cell signaling networks (DiFrisco et al., 2020). In support of this hypothesis, a recent comparative study of gene expression in turtles and chicken found that, in the notochord, somites, and neural tube, Shh and genes involved in the Shh cascade are more highly conserved than other genes (Fujimoto et al., 2022). A key difference with many of the putative kernels, Hox genes, and other core regulators discovered in developmental genetics, is that individual signaling pathways are not specific to particular tissues or particular character identities. Across Metazoa, the same 7 intercellular signaling pathways are used, re-used, and combined to regulate multicellular development in diverse ways (TGF- $\beta$, Wnt, nuclear receptors, RTK, Notch/Delta, Hedgehog, and JAK/STAT) (Babonis and Martindale 2016). In signaling pathways, multiple varying inputs can be transduced through a cytosolic module that then sets up a wide variety of responses from the nucleus (Pires-daSilva and Sommer 2003). They are not based on linear sequential cascades but can instead branch at many steps of a transduction process, which contributes to the variety of developmental roles they can take in different tissues and species (Pires-daSilva and Sommer 2003). These broad differences predict different patterns of evolutionary conservation. Instead of the hierarchical model of GRN evolution where upstream regulatory genes are more conserved than downstream effector genes (Davidson and Erwin 2006)_an early conservation model_one can expect a "bow-tie" pattern where the signaling pathway is conserved through varying gene-regulatory inputs and outputs. Further comparative studies are needed before this hypothesis can be more rigorously evaluated.

The causal profile of ChIMs-active modularity, complex organization, interdependence and necessity, and non-redundancy-implies that they should be more entrenched and conserved in evolution than their upstream inputs and downstream outputs. If BpIMs share much of the same causal profile, as exemplified by the notochord signaling system, it can potentially explain the remarkable conservatism of the signaling mechanism as well as the morphological hourglass pattern resulting from it.

When a key feature of a major phyletic group gets secondarily lost, conversely, one can expect some underlying difference at the level of the BpIM or the causal profile and developmental dependencies that would otherwise keep it entrenched. Tunicates, a chordate sister group of the vertebrates, seem to lack many of the developmental constraints characterizing the vertebrate body plan, including some losses of the notochord and associated structures. They are thus a potentially useful test case for the above hypotheses about body plan identity and its developmental basis. The dispensability of the notochord in some tunicates suggests that the BpIM and entrenched body plan in our developmental-mechanistic sense do not lie at the level of "chordates," but rather at the level of vertebrates or possibly even a paraphyletic group including the vertebrates.

\subsection{Secondary losses of the notochord}

One group of Tunicates, ascidians (sea squirts), has a metamorphic life cycle in which freeswimming tadpole-like larvae tend to exhibit chordate phylotypic features, but are followed by a derived sessile adult stage that has very little morphological resemblance to vertebrates. Some adult ascidians form colonies that can reproduce adults via asexual budding, bypassing the "phylotypic" tadpole stage altogether (Lemaire et al., 2008). Most ascidian larvae are short-lived and morphologically very simple compared to other chordates, including the more basal but more 
vertebrate-like cephalochordates (amphioxus) (Delsuc et al., 2006). Ascidian larvae seem to be largely adapted for dispersal to find appropriate benthic attachment sites, and for this they rely on axial tail muscles and the notochord. Some ascidian families (Styelidae and Molgulidae) include species that have simplified the larval stage even further. They disperse passively via ocean currents, and have lost the notochord entirely along with the tail, muscles, and most larval sensory organs (Lemaire et al., 2008; Huber et al., 2000). Losses of the notochord can also be found in another tunicate group, thaliaceans, with larval stages similar to ascidians but free-floating pelagic adults (Piette et al., 2015).

Given how entrenched and developmentally essential notochord signaling is in vertebrate model organisms, how should these secondary losses be understood? Although the molecular mechanisms of tunicate notochord development are not well known, two broad hypotheses may be helpful for guiding further investigation. First, in early chordate evolution the notochord was likely more of an ordinary character with an adaptive function (i.e., support for swimming) than a deeply entrenched body plan character. Many of the signaling functions that entrench the notochord with developmental functions were probably added later in vertebrate and gnathostome evolution. Its presence or absence in early chordates would then depend more on ecological rather than developmental conditions, which fits the pattern of repeated independent losses in the ascidians (Huber et al., 2000). Second, even if the primitive condition is one in which major characters already depend developmentally on the notochord, complementary body plan simplifications may have eliminated these dependencies and thereby enabled adaptive losses of the notochord. For example, the more basal amphioxus has somites, but tunicates lost them (Ruppert 2005), and the non-motile ascidian larvae have also lost sensory neurons and the swimming tail (Lemaire et al., 2008).

Although the notochord is homologous across all chordates, it evidently has a very different role and status in the body plan between tunicates and other chordates. This highlights how, from a developmental point of view, a body plan is not just a set of homologies, but is rather a set of homologies that is mechanistically integrated and entrenched in characteristic ways. Because this type of entrenchment likely arises in evolution through a process of stepwise accretion, the first appearance of the character can pre-date its evolutionary-developmental entrenchment (see Valentine 1975; 1977). This suggests that the body plan that includes the notochord, and the corresponding BpIM that includes notochord signaling, are not to be identified at the level of "chordates," but rather vertebrates. Whether that body plan is shared by certain tunicates, yielding a paraphyletic body plan, remains an open question for developmental investigation.

\section{Non-chordate body plans}

Having explicated the conceptual model of BpIMs from Section 4.2 in terms of vertebrate model organisms, the question naturally arises how generalizable this model is to other major clades. Do other clades have body plans with underlying BpIMs possessing the same causal profile and general architecture-i.e., entrenched signaling interactions across pre-individualized embryonic structures in mid-development-or are there important differences? While the lack of extensive comparative and experimental data for many clades make it impossible to give a definitive answer 
to this question, it is valuable nonetheless to begin assessing the situation based on more wellstudied clades in order to sketch directions for further comparative research on body plans.

The most thoroughly studied phylum outside of chordates is the arthropods (Euarthropoda, including Hexapods, Crustaceans, Myriapods, and Chelicerates), particularly certain holometabolous insects like Drosophila. Arthropods have a metameric body organization with conspicuous segmentation of the exoskeleton as well as segmentation of the internal organs, at least during development, comprising the neural, muscular, circulatory, and excretory systems. A segmented body organization appears to contribute to high evolvability especially among insects, which are one of the most species-diverse clades in existence (Mora et al., 2011). Among arthropods, the most morphologically conserved stage (or phylotypic stage) is the "segmented germ band," where segmental boundaries are being defined by the activity of a conserved mechanism known as the segment polarity network (SPN) (Patel 1994; Raff 1996; Peel et al., 2005). The SPN has been proposed as the only plausible candidate for a "conserved ChIN" for arthropod segments (Wagner 2014; Auman and Chipman 2017) -though it can readily be viewed as a body plan identity mechanism given that the SPN is active throughout the embryonic trunk and underlies its segmental body organization. As with vertebrates, these comparative morphological and mechanistic findings have been bolstered by large-scale transcriptomic studies that found that gene expression divergence is minimized during the extended germ band stage among a sample of Drosophila species (Kalinka et al., 2010).

Most of what is known about arthropod segmentation is centered on insects, where there is already significant diversity in the segmentation cascade preceding activation of the SPN. Within insects, the major contrast is between sequential versus simultaneous modes of segmentation. In the ancestral, sequential mode, segments are generated in a posterior region of the embryo called the Segment Addition Zone, where spatial periodicities are generated via a clock and wavefront process analogous to the vertebrate segmentation clock (Oates et al., 2012; Clark et al., 2019; DiFrisco and Jaeger 2021). Sequential segmentation is gradual and usually accompanied by growth and elongation of the embryo, as in vertebrates. In contrast, dipterans like Drosophila and other holometabolous insects have evolved to segment the entire larva nearly simultaneously on the basis of aperiodic gradients. Simultaneous segmentation occurs predominantly before gastrulation and without embryonic growth, in a syncytial blastoderm. Intermediate forms between these modes of segmentation exist, e.g. in Hemiptera and certain beetles (Chipman and Edgecombe 2019) and evolutionary transformations of sequential to simultaneous segmentation have occurred repeatedly (DiFrisco and Jaeger 2021). Among noninsect arthropods, segmentation processes appear to be more diverse still, despite converging nonetheless on the segmented germ band stage (Peel et al., 2005).

The molecular mechanisms driving segmentation are most well-studied in Drosophila. Starting from gradients established by maternal factors deposited in the egg, the segmentation cascade runs through a series of regulatory networks-gap genes, pair-rule genes, and the segment polarity network - with each providing patterning input to the next and sharpening the subdivisions of the embryo. Later in the segmentation cascade, axial identities are conferred by Hox genes and arthropod groups diverge again (Peel et al., 2005). Unlike in simultaneous segmentation, many gap genes in sequential segmentation have different functions and expression domains (Peel 2004; Peel et al., 2005) and do not appear to play a significant role in directing pair-rule gene expression, though they do pattern Hox gene expression (Clark et al., 2019). In non-insect 
arthropods there is limited information on gap genes (Auman and Chipman 2017). In line with the hourglass pattern, the role of the pair-rule network in arthropod segmentation appears to be relatively more conserved than that of gap genes, with the major differences in expression patterns deriving from morphological differences between sequential and simultaneous segmenting embryos as well as associated differences in upstream regulatory inputs (cell-cell signaling pathways versus transcription factors diffusing freely in a syncytium) (Damen 2007).

The segment polarity network (SPN) operates on domains defined by pair-rule genes to demarcate and give anterior-posterior polarity to ectodermal embryonic structures known as "parasegments" (See Fig 2). Parasegments are the basic developmental units of the arthropod embryo, organizing the major organ systems into compartments that also correspond to Hox gene expression domains (Damen 2007). Like vertebrate somites and vertebrae, they are offset from the eventual cuticular segments, which form protective exoskeletal articulations between parasegments (Clark et al., 2019). The boundaries between parasegments are maintained and refined by cross-regulatory interactions in the SPN, which consists of cell-cell signaling pathways - wingless (wg) and hedgehog (hh), homologues of Wnt and Shh in vertebrates-and transcription factors (e.g., engrailed (en), cubitus interruptus (ci), and gooseberry (gsb)) (Damen 2007). In the SPN's main mechanism of action, the posterior compartment of each parasegmental unit expresses $w g$, which activates $h h$ in cells of the anterior compartment of the adjacent parasegment. This anterior row of cells then expresses en, which stabilizes its own expression, as well as $h h$, which in turn stabilizes expression of $w g$ in the neighboring cell in an autoregulatory loop (see Fig 2; Chipman 2020).

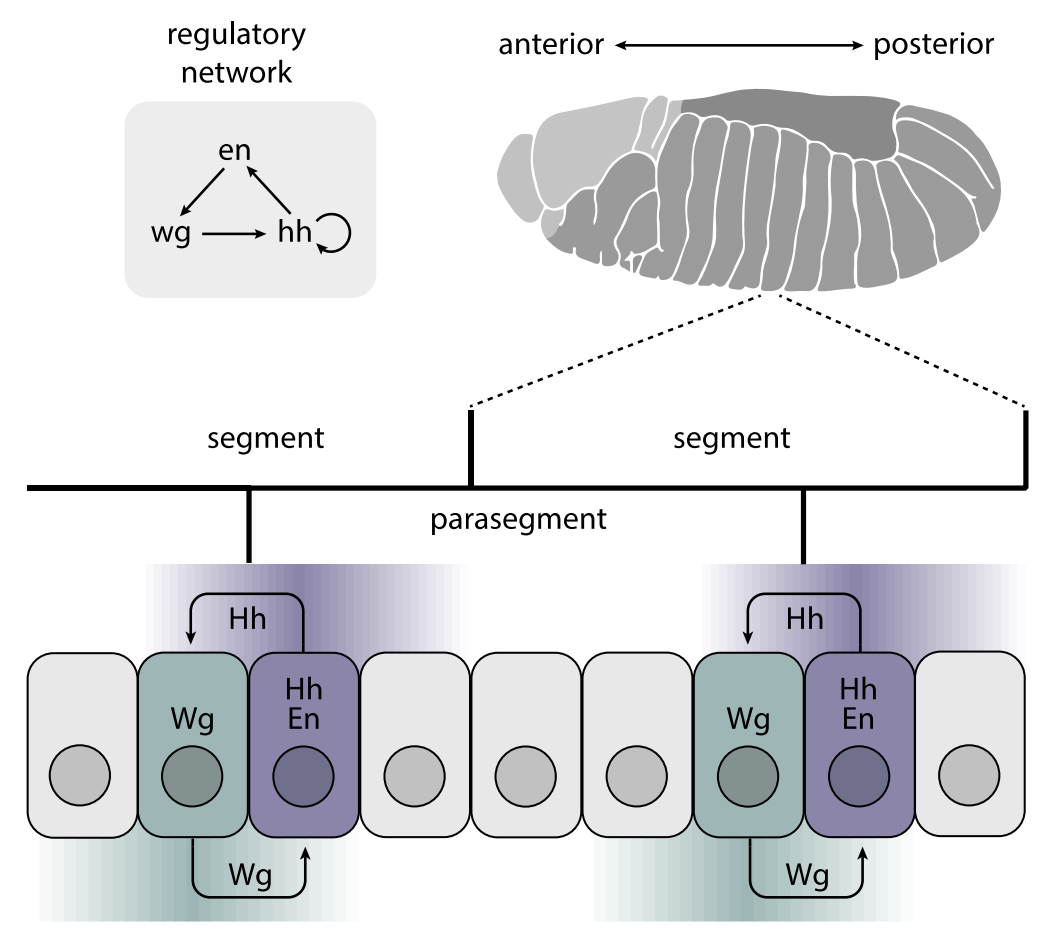

Fig 2. A simplified illustration of the segment polarity network (SPN) showing the basic positive feedback circuit, its implementation in forming parasegmental boundaries, and the phase shift of 
parasegments and segments. The segmented germband stage has been identified as an arthropod "phylotypic stage" (shown here in Drosophila).

These signaling cells along the parasegmental boundary function as an "organizer" with cascading effects on crucial subsequent developmental processes, including the differentiation of virtually all organ primordia, inductive signaling across germ layers, as well as major morphogenetic events such as germband retraction and dorsal closure (Galis et al., 2002). SPN signaling influences segmentation and differentiation of very many ectodermal precursor cells including neuroblasts, sensory precursor cells, and imaginal discs. Defective segmentation of the ectoderm disrupts segmentation of mesodermal heart and muscle primordia, which is directed by inductive signals from the ectoderm (Galis et al., 2002; Clark et al., 2019), as well as subsequent induction of endodermal structures (Bienz, 1997). Severe and/or lethal abnormalities in these key processes follow from both loss of function and hypomorphic mutations of most SPN genes (Galis et al., 2002), which indicates a mechanistic explanation of the evolutionary conservation of the SPN as well as the segmented germ band stage in arthropods.

The features of the SPN just reviewed conform to the general model of BpIMs proposed earlier, showing that the latter is not merely specific to vertebrates. The SPN is a BpIM that operates by inductive signaling across pre-individualized embryonic structures (parasegments) that are destined to develop and differentiated into modular, individualized characters. Although the role of induction across germ layers is not as central in arthropods as in vertebrates, the signaling interactions of the SPN similarly provide a transient global integration of the embryo that is necessary for providing embryonic precursors the competence for complex differentiation and organogenesis. The SPN also displays the proposed causal profile of BpIMs. The autoregulatory loop involving $w g$ and $h h$ signaling exhibits active modularity in the sense that it robustly reinforces its own dynamics and inhibits alternative regulatory states, and also clearly exhibits complex organization (von Dassow et al., 2000; Zañudo et al., 2017; Chipman, 2020). Components of the SPN are individually and collectively necessary for the organization of the body plan, as seen by the lethal effects of mutations, and genetic redundancy is low though not absent completely (Galis et al., 2002). The reliance on intercellular signal transduction pathways ( $w g$ and $h h$ ), modulated by intracellular networks of transcription factors, also suggests the SPN exhibits the bow-tie conservation pattern of signaling mechanisms, though confirmation of this feature would require more thorough investigation. Together, this causal profile begins to account for how the SPN can function as an entrenched, highly conserved BpIM for the arthropod body plan.

Interestingly, one comparative gene expression study on Drosophila found that expression divergence is minimized during pupation, indicating an additional hourglass pattern beyond the larval segmented germband stage (Artieri and Singh, 2010). This supports the classic study of Woskressensky (1928) on Drosophila, which found that vulnerability to induced mortality via Xrays was highest during pupation. Drosophila and other holometabolous insects undergo two phases of extensive organogenesis: one during larval embryogenesis and another during pupal metamorphosis, where many larval organs and tissues are re-specified and long-range interactions occur in the imaginal discs. Analogously to body plan characters like the notochord, the pupal stage is ecologically inert-a "purely developmental component of the holometabolous life cycle" (Rolff et al., 2019). It is natural to infer the existence of an adult body plan as well as a BpIM in addition to the larval body plan and larval BpIM (i.e., the SPN). Further study would be 
needed to confirm whether pupal organogenesis exhibits the proposed features of BpIMs, and to establish the phylogenetic scope of this hypothetical additional BpIM, given that the extent of body reorganization during metamorphosis differs within the holometabolous insects (Rolff et al., 2019). Less dramatic metamorphic life cycles such as that of anurans are unlikely to have multiple body plans in the robust developmental-mechanistic sense, whereas this is more likely in the metamorphosis of other non-insects such as sea urchins (Raff, 1996). Thus one cannot necessarily infer distinct sequential body plans merely from the designation of some life cycle as "metamorphic" (Bishop et al., 2006).

A number of implications would follow from the existence of multiple body plans and BpIMs within the same metamorphic life cycle, as in holometaboly. First, it is a further counterargument to the assumption that distinct metazoan body plans exist only at the taxonomic level of phyla. It also indirectly highlights how the relevant notion of body plan is not a purely phylogenetic one, as it is not clear how the latter could avoid attributing body plan novelty to every distinct clade. Given that holometabolous insects probably emerged during the Carboniferous, their body plan novelty goes against the common claim — linked to the "body plans as phyla" idea-that animal body plans were all definitively established by the Cambrian. At the same time, it does not contravene the phenomenon of body plan stasis: holometaboly involves an additional body plan evolving in a life cycle having one already, rather than a transformation of one entrenched body plan into another. Another implication is that BpIMs in metamorphic life cycles may play the biological role of individualization, as ChIMs do for characters within an organism, but for modular phases in a life cycle. Complete metamorphosis enables a body plan to escape the developmental constraints imposed by the ancestral adult-like body plan, allowing a decoupling of growth and differentiation and extensive functional specialization of different stages (Rolff et al., 2019; Istock, 1966). This can even lead to secondary losses of entire body plans, which has been observed in some pedogenic insects (McMahon and Hayward, 2016), and frequently in metagenic marine invertebrates (Moran, 1994). Finally, the complete metamorphosis that occurs during pupation differs from the BpIMs considered so far in being directed by hormones from the neural-endocrine system, which are products of late development of the preceding larval or juvenile stage. In complete metamorphosis, then, the second body plan may not require repeating the whole stereotyped sequence of early, mid, and late development (see Section 4.1). That whole sequence may be iterated in metagenetic life cycles, which involve alternations between asexual and sexual generations with distinct organizational forms (or body plans) (Fusco and Minelli 2019). In complete metamorphosis, by contrast, the stages of pupation and beyond may proceed without an early development phase.

Outside of arthropods, comparative transcriptomic studies have broadly confirmed that phylotypic stages previously identified correspond to periods of mid-development where expression divergence is minimized (Levin et al., 2016; Irie and Kuratani, 2011). These include the ventral enclosure stage for nematodes (Levin et al., 2012), the late trochophore stage for annelids (Fisher et al., 2010), and the stage where embryonic and adult pharynxes are joined for flatworms (Martín-Durán and Egger 2012). Levin et al., (2016), sampling 10 species from various phyla, found that gene expression during the transition between early and late development was significantly enriched for signaling pathways such as Wnt and Notch, indicating the activity of signaling networks that may turn out to constitute BpIMs in the sense proposed here. 
Beyond the metazoa, some studies have detected transcriptomic hourglasses in fungi and plants, but curiously, these appear not to be neatly reflected in hourglass patterns of morphological conservation (Drost et al., 2015; Drost et al., 2017). It is tempting to speculate that this morphological variability is enabled in plants by the fact that organogenesis occurs without inductive signaling across germ layers, and also continues after embryonic development in an iterative fashion (as contrasted with near-simultaneous organogenesis in animals). The biological reason for a convergent transcriptomic hourglass across kingdoms is far from clear, however, as are the factors that allow its decoupling from morphological hourglass patterns. These and many other puzzles stand as open challenges to an empirical research program on the evo-devo of body plans.

\section{Discussion: body plans beyond typology and genealogy}

In this paper, we have proposed an abstract mechanistic model of body plan identity that can begin to address macroevolutionary phenomena such as body plan stasis while also serving as a framework for body plans as an organizing concept of comparative biology. The proposed model of body plan identity mechanisms (BpIMs) has a clear kinship with the family of hourglass models of development (Sander 1983; Duboule 1994; Raff 1996) while also providing it with a more precise basis in current developmental biology and genetics.

Our central hypothesis is that BpIMs have the same basic architecture and causal profile as character identity mechanisms for organs-i.e., cell-cell signaling networks characterized by active modularity, complex organization, collective necessity, and non-redundancy (DiFrisco et al., 2020). In BpIMs, however, the tissue domains covered by these signaling mechanisms are weakly individualized, transient embryonic structures, often from distinct germ layers, that are destined to differentiate into distinct individualized characters and organs under the control of different ChIMs. The activity of a BpIM results in a transient long-range integration of the embryo, detectable morphologically as a phylotypic stage. This stage and the underlying BpIM are especially refractory to evolutionary change due to entrenched interdependencies between the parts as well as entrenched dependencies of later developmental stages on the organizing activity of the BplM.

It is important to recognize that the biological phenomena targeted by this model are exceedingly complex and demand extensive comparative data on ontogenetic diversity to be validated. In this situation, what models can offer will be incomplete and partially idealized, but such models can nonetheless clear the way toward better theories (Wimsatt 2007; DiFrisco et al., 2020). They do this by generating hypotheses that, when tested, may ultimately point toward more complete, supplementary, or even alternative models. Revision or supplementation of the BpIM model seems likely to come from more extensive incorporation of comparative mechanistic data.

While recognizing the provisional status of the model, we can nonetheless already extract some general implications for the nature and status of body plans. A great source of confusion surrounding the concept of body plans is that it tends to be overburdened with incompatible demands (Budd and Jensen, 2000; Wray and Strathmann, 2002): (1) it should provide some wideranging generalizations about phenotypic organization based on developmental mechanisms, and at the same time, (2) its extension should correspond to a monophyletic clade, or the 
Linnaean rank of phylum specifically. It should not be surprising that metazoan evolution is too historically contingent and diverse to neatly conform to these requirements. One response to this situation is to discard the body plan concept along with the archetype as a remnant of outdated typological thinking (Scholtz 2004; 2010; Ghiselin 2005). An alternative response is to differentiate the general notion of body plans into phylogenetic and developmental-mechanistic versions. In the framework of phylogenetic systematics, a body plan can be viewed as a set of characters that arise in the stem lineage of a clade, including a combination of ancestral plesiomorphies and derived apomorphies. This is sometimes referred to as the "ground plan" or "ground pattern" (Hennig, 1965; Ax, 1984; Yeates, 1995; Scholtz, 2004). In contrast, according to the developmental-mechanistic view, a body plan is a set of entrenched character identities that are integrated by the activity of the same BpIM. These character identities comprise the (sub)set of ground plan characters that meets the additional, more restrictive developmental conditions of entrenchment and mechanistic integration. The difference between the two concepts can be appreciated by the fact that the part of the vertebrate BpIM we described-the entrenched notochord signaling system - is not shared by all chordates, despite the fact that the notochord is a chordate synapomorphy. This is not only because some chordates have secondarily lost the notochord, but also because some chordates have a notochord that is not entrenched as a body plan character.

The basic investigative procedure for identifying body plans in our proposed developmental sense would run as follows. First, start with some monophyletic group whose component species are reasonably hypothesized to share a distinctive body organization. Next, attempt to identify phylotypic stage(s), body plan identities, and BpIM(s) within the group using a combination of comparative morphology, embryology, genetics, and experimental methods. Finally, if these features can be identified, revise the initial grouping that was based on the phylogenetic ground plan to yield the group that shares the same developmental body plan-which may or may not be monophyletic. In this approach, the developmental body plan concept initially relies on phylogenetic classification while ultimately branching off into an investigative goal other than mapping phylogeny. The main advantage of differentiating the body plan concept along these lines, rather than eliminating it or reducing it to phylogenetic ground plans, is that we do not discard the mechanisms and generalizations that are informative about phenotypic organization only for the sake of conceptual consistency with monophyly.

Among evolutionary biologists, the notion of body plans is sometimes negatively associated with a regression to "typological thinking" (Mayr, 1982; Scholtz, 2004; 2010; Ghiselin, 2005; Amundson, 2005). According to its critics, typological thinking takes categories like "species," "animal," or "body plan" as ideal types that possess essentialistic criteria of membership and that ground exceptionless laws of nature (Mayr, 1959; 1982; Dobzhansky, 1950; Ghiselin, 2005; Hull, $1967 ; 1978)$. This is understood to be incompatible with transformation from one species to another, with intra-type variation, historical contingency, and other features of Darwinian evolution. The standard contrast to typological thinking is "population thinking" (Mayr 1982), which rejects essentialism and takes variation and evolutionary change as the norm. For the body plan concept, a more relevant contrast would be "genealogical thinking" or "tree thinking" (Sarasin 2009; Scholtz 2010). Genealogical thinking takes biological classification to be based exclusively on phylogeny and transformation series between "types," which effectively "dissolves the type concept" (Scholtz 2010). 
Although we cannot give a systematic discussion of this general issue here-see Amundson (2005); Love (2008); Ereshefsky (2012) - it is necessary to specifically point out how the mechanistic body plan concept evades what is problematic about typological thinking (as opposed to what is mere guilt by association). Body plans are types, but conceptions of types or natural kinds have been developed that are non-essentialist and fully compatible with evolutionary transformation (Riedl 1978; Boyd 1999; Griffiths 1999; Wilson 1999; Brigandt 2009; Wagner and Tomlinson 2021). General statements about body plans-e.g., "in vertebrates, differentiation of somitic tissues depends on gradients of Shh secreted by the notochord"-are not immutable laws of nature. They are contingent, inductive generalizations that are subject to exceptions. Crucially, the possibility of exceptions does not render these generalizations trivial or uninformative: the entrenched developmental dependencies captured by BpIMs are part of the explanation for why those generalizations hold when they do, and help us to understand why and how exceptions arise. Chordates that lost the notochord seem to have experienced body plan simplifications that are complementary according to our BpIM model (e.g., loss of somites, sensory neurons, and/or swimmings tails), leading to a weaker burden on the notochord. So, it is not the type per se ("vertebrate body plan") that is doing the explanatory work of supporting generalizations about body plans. Both the generalizations and the type are grounded on underlying causal dependencies in development, which are contingent products of evolution as well as contingent influences on further evolution.

The available alternatives to typological thinking-population thinking and genealogical thinking-have, by contrast, limited prospects to illuminate the phenomenon of entrenched character configurations. The evolution of body plans is not an investigatable problem for the focal disciplines of population thinking (population genetics and quantitative genetics) (Riedl 1978). With phylogenetic ground plans, although they are undoubtedly useful for describing patterns of conservation and change, they cannot explain why those patterns of conservation obtain. Ground plans, exhibited in all and only monophyletic groups, can also easily fail to capture informative generalizations about body organization and conservation. For example, from a phylogenetic perspective, transmissible cancer cells derived from mammals would be "single-celled mammals" whose ground plan would be that of the common ancestor of all mammals (or the stem species of any other more or less inclusive monophylum). Differentiating such radically distinct forms of phenotypic organization requires a body plan concept that is based on more stringent biological criteria than genealogical relatedness alone.

Body plans, in our envisioned approach, are phenomena caused by entrenchment in the evolution of developmental complexity. Like major transitions in individuality or the evolution of sexuality, these phenomena can occur under specific circumstances, but they are neither predestined by evolution nor guaranteed merely in virtue of the definition of terms (like "phylum" or "clade"). Whether some evolved group exhibits a distinctive body plan is a biological hypothesis to be investigated, as is the extent of conservation for BpIMs. In this approach, the existence and nature of body plans is transformed from an a priori issue concerning one's metaphysics of evolution into an empirical issue that is open to discovery. 


\section{References}

Almeida, N. Chung, M.W.H. Drudi, E.M. Engquist, E.N. Hamrud, E. Isaacson, A. Tsang, V.S.K. Watt, F.M. Spagnoli, F.M. (2021). Employing core regulatory circuits to define cell identity, The EMBO Journal 40(10): e106785.

Amundson, R. (2005). The Changing Role of the Embryo in Evolutionary Thought: Roots of EvoDevo. Cambridge: Cambridge University Press.

Ando T, Semba K, Suda H, Sei A, Mizuta H, Araki M, Abe K, Imai K, Nakagata N, Araki K, Yamamura K (2011) The floor plate is sufficient for development of the sclerotome and spine without the notochord. Mech Dev 128:129-140.

Annona, G, Holland, N. D., D'Aniello, S. (2015). Evolution of the notochord. EvoDevo 6:30

Arendt, D., Musser, J.M., Baker, C.V.H., Bergman, A., Cepko, C., Erwin, D.H., Pavlicev, M., Schlosser, G., Widder, S., Laubichler, M.D., Wagner, G.P. (2016). The origin and evolution of cell types. Nature Reviews Genetics 17(12): 744-757.

Arthur, W. 1988. A Theory of the Evolution of Development. John Wiley \& Sons, Chichester.

Artieri, C. G., \& Singh, R. S. (2010). Molecular evidence for increased regulatory conservation during metamorphosis, and against deleterious cascading effects of hybrid breakdown in Drosophila. BMC Biology 8: 26.

Auman, T., \& A. D. Chipman. (2017). The evolution of gene regulatory networks that define arthropod body plans. Integrative and Comparative Biology 57(3): 523-532.

Ax, P. 1984. Das Phylogenetische System. Stuttgart, Gustav Fischer.

Babonis, L. S., \& Martindale, M. Q. (2016). Phylogenetic evidence for the modular evolution of metazoan signalling pathways. Philosophical Transactions of the Royal Society B. 372: 20150477.

Bienz, M. (1997) Endoderm induction in Drosophila: the nuclear targets of the inducing signal. Curr. Opin. Genet. Dev. 7, 683-688

Bishop, C.D., Erezyilmaz, D.F., Flatt, T., Georgiou, C.D., Hadfield, M.G., Heyland, A., Hodin, J., Jacobs, M.W., Maslakova, S.A., Pires, A., Reitzel, A.M., Santagata, S., Tanaka, K., Youson, J.H. (2006). What is metamorphosis? Integrative and Comparative Biology 46: 655-661.

Boyd, R. (1999). Homeostasis, species, and higher taxa. In R. A. Wilson (Ed.) Species: New Interdisciplinary Essays, MIT Press, pp. 141-85.

Brigandt, I. (2009). Natural Kinds in Evolution and Systematics: Metaphysical and Epistemological Considerations. Acta Biotheoretica 57: 77-97.

Briscoe, J, and Small, S. (2015). Morphogen rules: design principles of gradient-mediated embryo patterning. Development 142(23): 3996-4009.

Budd, G. E., \& Jensen, S. (2000). A critical reappraisal of the fossil record of the bilaterian phyla. Biological Reviews 75: 253-295.

Clark, E., Peel, A.D., \& Akam, M. (2019) Arthropod Segmentation. Development 146: dev170480.

Cleaver, O., \& Krieg, P. A. (1998). VEGF mediates angioblast migration during development of the dorsal aorta in Xenopus. Development 125: 3905-3914.

Corallo, D., Trapani, V., \& Bonaldo, P. (2015). The notochord: structure and functions. Cellular and Molecular Life Sciences 72: 2989-3008. 
Chipman A. D., Edgecombe G. D. (2019). Developing an integrated understanding of the evolution of arthropod segmentation using fossils and evo-devo. Proc. R. Soc. B 286, 20191881.

Chipman, A. D. (2020). The evolution of the gene regulatory networks patterning the Drosophila Blastoderm. In I. Peter (Ed.) Current Topics in Developmental Biology vol. 139, Gene Regulatory Networks, pp. 297-324. Academic Press: Cambridge, MA.

Damen, W. G. M. (2007). Evolutionary Conservation and Divergence of the Segmentation Process in Arthropods. Developmental Dynamics 236: 1379-1391.

Deutsch, J. (2005). Hox and wings. BioEssays 27(7): 673-675.

Delsuc, F., Brinkmann, H., Chourrout, D., and Philippe, H. (2006). Tunicates and not cephalochordates are the closest living relatives of vertebrates. Nature 439, 965-968.

Davidson, E. H., \& Erwin, D. H., (2006). Gene Regulatory Networks and the Evolution of Animal Body Plans. Science 311: 796-800.

DiFrisco, J, Love, A.C., \& Wagner, G.P. (2020). Character identity mechanisms: a conceptual model for comparative-mechanistic biology. Biology \& Philosophy 35(4): 44.

DiFrisco, J., \& Jaeger, J. (2021). Homology of process: developmental dynamics in comparative biology. Interface Focus 11: 20210007.

DiFrisco, J, Wagner, G.P., \& Love, A.C., (in press). Reframing Research on Evolutionary Novelty and Co-Option: Character Identity Mechanisms versus Deep Homology.

De Beer, Gavin. (1954). Embryos and Ancestors. Revised Ed. Oxford: Oxford University Press.

Dobzhansky, T. (1950). "Human diversity and adaptation." Cold Spring Harbor Symposia on Quantitative Biology 15: 385-400.

Drost, H.-G., Gabel, A., Grosse, I., \& Quint, M. (2015). Evidence for Active Maintenance of Phylotranscriptomic Hourglass Patterns in Animal and Plant Embryogenesis. Mol. Biol. Evol. 32(5): 1221-1231.

Drost, H.-G., Janitza, P., Grosse, I., \& Quint, M. (2017). Cross-kingdom comparison of the developmental hourglass. Current Opinion in Genetics \& Development 45: 69-75.

Duboule, D. (1994). Temporal colinearity and the phylotypic progression: a basis for the stability of a vertebrate Bauplan and the evolution of morphologies through heterochrony. Development, 120 Supplement: 135-142.

Dumont, D. J., Fong, G.-H., Puri, M. C., Gradwohl, G., Alitalo, K. and Breitman, M. L. (1995). Vascularization of the mouse embryo: a study of flk-1, tek, tie, and vascular endothelial growth factor expression during development. Dev. Dyn. 203, 80-92.

Ebisuya, M., \& Briscoe, J. (2018). What does time mean in development? Development 145: dev164368.

Eldredge, N. and Cracraft, J. (1980). Phylogenetic Patterns and the Evolutionary Process: Method and Theory in Comparative Biology. New York: Columbia University Press.

Elinson R. P. (1987). Change in developmental patterns: embryos of amphibians with large eggs. In: Raff RA, Raff EC (Eds.) Development as an evolutionary process. Alan R. Liss, New York, pp 1-21.

Ereshefsky, M. (2012). Homology thinking. Biology \& Philosophy 27: 381-400.

Erwin, D. H., J. W. Valentine, and J. J. Sepkoski Jr. 1987. A comparative study of diversification events: The early Paleozoic versus the Mesozoic. Evolution 41: 1177-86. 
Favarolo, M. B., \& López, S. L. (2018). Notch signaling in the division of germ layers in bilaterian embryos. Mechanisms of Development 154: 122-144.

Fischer, A. H., Henrich, T. \& Arendt, D. (2010). The normal development of Platynereis dumerilii (Nereididae, Annelida). Front. Zool. 7, 31

Fujimoto, S., Yamanaka, K., Tanegashima, C., Nishimura, O., Kuraku, S., Kuratani, S., \& Irie, N. (2022). Measuring potential effects of developmental burden associated with the vertebrate notochord. J Exp Zool B (Mol Dev Evol) 338, 129-136.

Fusco, G., \& Minelli, A. (2019). The biology of reproduction. Cambridge, UK: Cambridge University Press.

Hall, B. (1992). Evolutionary developmental biology. 2nd edition, London et al.: Chapman and Hall.

Galis, F., and Metz, J. A. J. (2001). Testing the Vulnerability of the Phylotypic Stage: On Modularity and Evolutionary Conservation. J Exp Zool (Mol Dev Evol) 291: 195-204.

Galis, F., van Dooren, T. J. M., Metz, J. A. J. (2002). Conservation of the segmented germband stage: robustness or pleiotropy? TRENDS in Genetics 18(10): 504-509.

Ghiselin, M. T., (2005). Homology as a relation of correspondence between parts of individuals. Theory in Biosciences 124: 91-103.

Grapin-Botton, A., and Melton, D. A. (2000). Endoderm development from patterning to organogenesis. Trends in Genetics 16(3): 124-130.

Griffiths, P. E. (1999). Squaring the circle: Natural kinds with historical essences. In R. A. Wilson (Ed.) Species: New Interdisciplinary Essays, MIT Press, pp. 209-228.

Gualdi, R. et al.,. (1996) Hepatic specification of the gut endoderm in vitro: cell signaling and transcriptional control. Genes Dev. 10, 1670-1682

Haag, E. S. and True, J. R. (2018). Developmental System Drift. In L. Nuño de la Rosa and G. B. Müller (Eds.), Evolutionary Developmental Biology: A Reference Guide, Cham: Springer.

Hall, B. K. (1995). Homology and Embryonic Development. In M. K. Hecht, R. J. Maclntyre, \& M. T. Clegg (Eds.) Evolutionary Biology, vol 28. Springer, New York: pp. 1-37.

Hazkani-Covo, E., Wool, D., and Grauer, D. (2005). In Search of the Vertebrate Phylotypic Stage: A Molecular Examination of the Developmental Hourglass Model and von Baer's Third Law. J. Exp. Zool. (Mol. Dev. Evol.) 304B:150-158.

Hebrok M., Kim S. K., Melton D. A. (1998) Notochord repression of endodermal Sonic hedgehog permits pancreas development. Genes \& Development 12:1705-1713

Hennig, W. 1965. Phylogenetic systematics. Annu. Rev. Entomol. 10: 97-116.

Hobert, O. (2008). Regulatory logic of neuronal diversity: Terminal selector genes and selector motifs. Proceedings of the National Academy of Sciences 105(51): 20067.

Hobert, O. (2011). Regulation of terminal differentiation programs in the nervous system. Annual Review of Cell and Developmental Biology 27: 681-696.

Huber, J. L., da Silva, K. B., Bates, W. R., \& Swalla, B. J. (2000). The evolution of anural larvae in molgulid ascidians. Seminars in Cell \& Developmental Biology 11: 419-426.

Hull, D. L. (1967). The metaphysics of evolution. British Journal for the History of Science 3(4): 309-337.

Hull, D. L. (1978). A matter of individuality. Philosophy of Science 45(3): 335-360.

Irie, N., and S. Kuratani. (2011). Comparative transcriptome analysis reveals vertebrate phylotypic period during organogenesis. Nature Communications 2: 248. 
Istock C. A. (1966). The evolution of complex life cycle phenomena: an ecological perspective. Evolution 67, 595-605.

Kalinka, A. T., Varga, K. M., Gerrard, D. T., Preibisch, S., Corcoran, D. L., Jarrells, J., Ohler, U., Bergman, C. M., \& Tomancak, P. (2010). Gene expression divergence recapitulates the developmental hourglass model. Nature 468: 811-814

Le Dréau, G., \& Martí, E. (2012). Dorsal-Ventral Patterning of the Neural Tube: A Tale of Three Signals. Developmental Neurobiology 72(12): 1471-1481.

Lemaire, P., Smith, W. C., \& Nishida, H. (2008). Ascidians and the plasticity of the chordate developmental program. Current Biology 18: R620-R631.

Levin, M., Anavy, L., Cole, A. G., Winter, E., Mostov, N., Khair, S., Senderovich, N., Kovalev, E., Silver, D. H., Feder, M., Fernandez-Valverde, S. L., Nakanishi, N., Simmons, D., Simakov, O., Larsson, T., Liu, S.-Y., Jerafi-Vider, A., Yaniv, K., Ryan, J. F., Martindale, M. Q., Rink, J. C., Arendt, D., Degnan, S. M., Degnan, B. M., Hashimshony, T., \& Yanai, I. (2016). The middevelopmental transition and the evolution of animal body plans. Nature 531: 637-641.

Levin, M., Hashimshony, T., Wagner, F., \& Yanai, I. (2012). Developmental Milestones Punctuate Gene Expression in the Caenorhabditis Embryo. Developmental Cell 22: 1101-1108.

Lewontin R. C. (1978). Adaptation. Scientific American 239(3): 212-231.

Long JH, Koob-Edmunds M, Sinwell B, Koob TJ. (2002). The notochord of hagfish Myxine glutinosa: visco-elastic properties and mechanical functions during steady swimming. J Exp Biol: 205: 3819-31.

Love, A. C. (2008). Typology Reconfigured: From the Metaphysics of Essentialism to the Epistemology of Representation. Acta Biotheoretica 57: 51-75.

Mayr, E. (1959). "Darwin and the evolutionary theory in biology." In B. J. Meggers (Ed.) Evolution and anthropology: A centennial appraisal. Washington, DC: Anthropological Society of Washington, pp. 1-10.

Mayr, E. (1970). Populations, Species, and Evolution: An Abridgment of Animal Species and Evolution. Cambridge, MA: Harvard University Press.

Mayr, E. (1982). The growth of biological thought. Cambridge, MA: Harvard University Press.

Martín-Durán, J. M. \& Egger, B. 2012. Developmental diversity in free-living flatworms. Evodevo $3,7$.

McKennan, K. Z., Wagner, G. P. \& Cooper, K. L. (2021). A developmental perspective of homology and evolutionary novelty. Curr. Topics in Developmental Biology 141: 1-38.

McMahon DP, Hayward A. (2016). Why grow up? A perspective on insect strategies to avoid metamorphosis. Ecol. Entomol. 41, 505-515.

Minelli, A., and Fusco, G. (2013). Homology. In K. Kampourakis (Ed.), The Philosophy of Biology: A Companion for Educators. Springer: Dordrecht, pp. 289-322.

Mora C, Tittensor DP, Adl S, Simpson AGB, Worm B. 2011. How Many Species Are There on Earth and in the Ocean? PLoS Biol 9, e1001127

Moran NA. 1994 Adaptation and constraint in the complex life cycles of animals. Annu. Rev. Ecol. Syst. 25, 573-600.

Nyhart, L. K. (1995). Biology Takes Form Animal Morphology and the German Universities, 18001900. University of Chicago Press.

Oates, AC, Morelli, AG, \& Ares, S. (2012). Patterning embryos with oscillations: structure, function and dynamics of the vertebrate segmentation clock. Development 139 (4), 625-639. 
Patel, N. H. (1994). Developmental Evolution: Insights from Studies of Insect Segmentation. Science 266: 581-590.

Peel, A. D. (2004). The evolution of arthropod segmentation mechanisms. BioEssays 26: 11081116.

Peel, A. D., Chipman, A. D., and Akam, M. (2005). Arthropod segmentation: beyond the Drosophila paradigm. Nature Reviews Genetics 6: 905-916.

Piasecka, B., Lichocki, P., Moretti, S., Bergmann, S., \& Robinson-Rechavi, M. (2013). The Hourglass and the Early Conservation Models-Co-Existing Patterns of Developmental Constraints in Vertebrates. PLoS Genetics, 9(4): e1003476.

Piette, J., \& Lemaire, P. (2015). Thaliaceans, the neglected pelagic relatives of ascidians: a developmental and evolutionary enigma. The Quarterly Review of Biology 90(2): 117-145.

Pires-daSilva A., \& Sommer, R. J. (2003). The evolution of signalling pathways in animal development. Nature Reviews Genetics 4: 39-49.

Raff, R. A. (1996). The shape of life: Genes, development, and the evolution of animal form. University of Chicago Press.

Remane, A. (1952). Die Grundlagen des natürlichen Systems, der vergleichenden Anatomie und der Phylogenetik. Akademische Verlagsgesellschaft Geest \& Portig K.G., Leipzig.

Ribes, V., Balaskas, N., Sasai, N., Cruz, C., Dessaud, E., Cayuso, J., Tozer, S., Yang, L. L., Novitch, B., Marti, E., and Briscoe, J. (2010). Distinct Sonic Hedgehog signaling dynamics specify floor plate and ventral neuronal progenitors in the vertebrate neural tube. Genes \& Development 24: 1186-1200.

Richardson, M. K., Hanken, J., Gooneratne, M. L., Pieau, C., Raynaud, A., Selwood, L., Wright, G. M. (1997). There is no highly conserved embryonic stage in the vertebrates: implications for current theories of evolution and development. Anat Embryol 196: 91-106.

Riedl, R. (1978). Order in Living Organisms: A Systems Analysis of Evolution, John Wiley \& Sons, Ltd, New York.

Rolff, J., Johnson, P. R., \& Reynolds, S. (2019). Complete metamorphosis of insects. Phil. Trans. R. Soc. B 374: 20190063.

Row, R. H., Tsotras, S. R., Goto, H., \& Martin, B. L. (2016). The zebrafish tailbud contains two independent populations of midline progenitor cells that maintain long-term germ layer plasticity and differentiate in response to local signaling cues. Development 143: 244-254.

Rupke, N. A. (2009). Richard Owen: Biology without Darwin. University of Chicago Press.

Ruppert, E. E. (2005). Key characters uniting hemichordates and chordates: homologies or homoplasies? Can. J. Zool. 83: 8-23.

Sander K. (1983). The evolution of patterning mechanisms: gleanings from insect embryogenesis and spermatogenesis. In Goodwin BC, Holder N, Wylie CC (Eds.), Development and Evolution, Cambridge University Press: pp. 137-159.

Sarasin, P. (2009). Darwin und Foucault. Suhrkamp Verlag, Frankfurt am Main.

Schmidt, K. \& Starck, J. M. (2011). Testing Evolutionary Hypotheses About the Phylotypic Period of Zebrafish. J. Exp. Zool. (Mol. Dev. Evol.) 316:319-329.

Scholtz, G. (2004). Baupläne versus ground patterns, phyla versus monophyla: aspects of patterns and processes in evolutionary developmental biology. In: Scholtz, G. (ed.). Evolutionary Developmental Biology of Crustacea. A. A. Balkema, Lisse, pp. 3-16.

Scholtz, G. (2010). Deconstructing morphology. Acta Zoologica 91: 44-63. 
Shubin, N., Tabin, C., Carroll, S. (1997). Fossils, genes and the evolution of animal limbs, Nature 388: 639-648.

Shubin, N., Tabin, C., Carroll, S. (2009). Deep homology and the origins of evolutionary novelty, Nature 457: 818-23.

Slack, J. M. W., Holland, P. W. H., and Graham, C. F. (1993). The zootype and the phylotypic stage. Nature 361: 490-492.

Stemple, D. L. (2005). Structure and function of the notochord: an essential organ for chordate development. Development 132: 2503-2512.

Tam, P.P.L., Gad, J.M., 2004. Gastrulation in the mouse embryo. In: Stern, C.D. (Ed.), Gastrulation. From Cells to Embryo. Cold Spring Harbor Laboratory Press, New York, pp. 233-262.

Tendolkar, A., Pomerantz, A.F., Heryanto, C., Shirk, P.D., Patel, N.H., \& Martin, A. (2021). Ultrabithorax is a micromanager of hindwing identity in butterflies and moths, Frontiers in Ecology and Evolution 9: 161.

Tripathi P, Guo Q, Wang Y, Coussens M, Liapis H, Jain S, Kuehn MR, Capecchi MR, Chen F (2010). Midline signaling regulates kidney positioning but not nephrogenesis through Shh. Dev Biol 340: 518-527.

True, J. R. and Haag, E. S. (2001). Developmental System Drift and Flexibility in Evolutionary Trajectories. Evolution \& Development 3(2): 109-19.

Valentine, J. W. (1975). Adaptive strategy and the origin of grades and ground-plans. American Zoologist 15: 391-404.

Valentine, J. W. (1977). General patterns of metazoan evolution. In A. Hallam (Ed.), Patterns of evolution, as illustrated by the fossil record. Elsevier, Amsterdam, 27-57.

Valentine, J. W. (2004). On the origin of phyla. University of Chicago Press, Chicago.

von Baer, K. E. (1828). Entwicklungsgeschichte der Thiere: Beobachtung und Reflexion. Bornträger, Königsberg.

von Dassow, G, Meir, E., Munro, E. M., \& Odell, G. M. (2000) The segment polarity network is a robust development module. Nature 406, 188-192

Wagner, G. P. (2007). The developmental genetics of homology. Nature Reviews Genetics 8(6) 473-479.

Wagner, G. P. (2014). Homology, Genes, and Evolutionary Innovation. Princeton University Press, Princeton, NJ.

Wagner, G. P., and Laubichler, M. D. (2004). Rupert Riedl and the Re-Synthesis of Evolutionary and Developmental Biology: Body Plans and Evolvability. J Exp Zool (Mol Dev Ecol) 302B: 92-102.

Wilson, R. A. (Ed.) (1999). Species: New Interdisciplinary Essays. Cambridge, MA: MIT Press.

Wimsatt, W. C. (1986). Developmental constraints, generative entrenchment and the innateacquired distinction. In W. Bechtel (Ed.), Integrating Scientific Disciplines, Martinus Nijhoff, Dordrecht, pp. 185-208.

Wimsatt, W. C. (2007). Re-engineering Philosophy for Limited Beings: Piecewise approximations to reality. Cambridge: Harvard University Press.

Wosskressensky N. M. (1928). Über die wirkung der röntgenbestrahlung auf das embryonale wachstum. Arch Entw Mech 113:447-461. 
Wray, G. and Strathmann R. (2002). Stasis, change, and functional constraint in the evolution of animal body plans, whatever they may be. Vie et Milieu, Observatoire Océanologique, 189199.

Yeates, DK. 1995. Groundplans and exemplars: paths to the tree of life. Cladistics 11: 343-357.

Zañudo JGT, Yang G, Albert, R. (2017). Structure-based control of complex networks with nonlinear dynamics. Proceedings of the National Academy of Sciences 114 (28), 7234-7239. 